Revista de Matemática: Teoría y Aplicaciones 2001 8(2) : 85-99

CIMPA - UCR - CCSS ISSN: 1409-2433

\title{
A METHOD FOR ANALYZING TURBULENCE MODELS
}

\author{
V. N. Grebenev*- B. B. Ilyushin ${ }^{\dagger}$
}

Received: 13 September 2000

\begin{abstract}
In this article we introduce a concept based on the differential constraints method to examine the closure procedure in Turbulence Models. We show how this concept may be applied to study the problem of interaction and mixing between two semiinfinite homogeneous turbulent flow fields of different scales.
\end{abstract}

Keywords: Turbulence models, closure procedure, differential constraints, invariant sets, third-order closure model, selfsimilar solution, asymptotic behavior.

\section{Resumen}

En este artículo introducimos un concepto basado en el método diferencial de restricciones para examinar el procedimiento de clausura en Modelos de Turbulencia. Mostramos como este concepto puede ser aplicado para estudiar el problema de interacción y mexclado entre dos campos de flujo turbulento homogéneo semi-infinito de diferentes escalas.

Palabras clave: Modelos de turbulencia, procedimiento de clausura, restricciones diferenciales, conjuntos invariantes, modelo de clausura de tercer orden, solución autosimilar, comportamiento asintótico.

\section{Introduction}

The method of differential constraints advanced by Yanenko [1], [2] for constructing explicit solutions to different kinds of nonlinear partial differential equations makes it possible to obtain the gradient-type algebraic expressions for unknown functions. This provides us a concept for examining the closure procedure for momentum equations in Turbulence

\footnotetext{
${ }^{*}$ Institute of Computational Technologies SB RAS, Novosibirsk 630090, Russia; E-Mail: vova@lchd.ict.nsc.ru

${ }^{\dagger}$ Institute of Thermophysics SB RAS, Novosibirsk 630090, Russia; E-Mail: ilyushin@itp.nsc.ru
} 
Models. Moreover, the method gives a reasonable tool for obtaining algebraic expressions. The exposition is demonstrated using example chosen from Free Turbulence Flows Theory. The analysis carried out in the article shows that a concept of differential constraints allows us to interpret algebraic expressions as the equations of invariant sets (manifolds) generated by corresponding differential equations.

Before proceeding to the mathematical contribution and for the sake of completeness we briefly inform the interested reader on the closure problem of Turbulence Models. The statistical moments method of turbulent flows structure description is based on the presentation of the sought quantities as a sum of mean values and turbulent fluctuations. Using the time-dependent Navier-Stokes equation and the procedure of averaging, we can derive transport equations for moments of arbitrary order. Since Fluid Mechanics Equations are nonlinear, any equation from the obtained ones, includes new unknown functions and corresponding transport equations can be derived for these unknowns too. However, the chain of the equations will remain unclosed because the introduction of new equations results in a quicker increase in the number of unknown functions than that of the number of equations. Thus, the exact turbulence model contains the infinite number of transport equations and generates the so-called closure problem. A general approach of closure strategy can be formulated as follows: cumulants of the order $1, \ldots, n$ are calculated from differential transport equations, the $(n+1)$-order cumulants describing the processes of turbulent diffusion in the $n$-order cumulants equations are calculated from the algebraic expressions derived from the corresponding transport equations and the $(n+2)$-order cumulants are considered to be zero, for details see [3]. Different closing approximations give different models. This general approach has been employed in [4] to obtain a closed model of turbulent transport that does not imply equality to zero of the fourth-order cumulants. The closure procedure was performed at the level of the fifth moments, i.e. the fifth-order cumulants were assumed to be equal to zero.

The plan of the article is as follows. In Section 2, we present a new third-order model of turbulence based on the above-mentioned approach to the classical problem about the dynamics of the shearless turbulence mixing layer. There are a considerable number of references connected with the use of third-order turbulence models. However, as a rule these models employ Millionshchikov's quasinormality hypothesis for the parametrization of diffusion processes in equations for triple correlations, that is according to this hypothesis, all cumulants of fourth and higher order can be negligible in comparison with the corresponding correlation functions. As a consequence, in the former case the triple-correlation equations are of the first-order without a dumping mechanism for triple correlations that leads (in some cases) to physically contradictory results [5] (e. g. the appearance of negative portions of the spectrum of the turbulent kinetic energy, [6], [7]). The approach proposed in [3], [4] allows us to overcome this obstacle; the technique also includes a physically reasonable way for constructing approximate algebraic parametrizations of higher moments.

Our next result is devoted to examining an algebraic expression for the triple correlation $\left\langle w^{3}\right\rangle$ which is generally used in second-order closure models by means of applying a concept of differential constraints to our third-order closure model. In Section 3 we show 
that the set of smooth functions:

$$
D=\left\{\left\langle w^{2}\right\rangle,\left\langle w^{3}\right\rangle, \hat{\tau}: \mathcal{H}^{1}\left(\left\langle w^{2}\right\rangle,\left\langle w^{3}\right\rangle, \hat{\tau}\right) \equiv\left\langle w^{3}\right\rangle+\delta \hat{\tau}\left\langle w^{2}\right\rangle\left\langle w^{2}\right\rangle_{z}=0\right\}
$$

is invariant under the flow generated by the differential equation for the triple correlation of the vertical velocity fluctuations. As a consequence, it is established that the differential constraint $\mathcal{H}^{1}$ coincides with the algebraic expression for $\left\langle w^{3}\right\rangle$ or the so-called tensorinvariant model[8].

In Section 4 we discuss the existence of selfsimilar solutions. It is wellknown [10] that if an operator admits invariant sets, then there exists a solution to an overdetermined system under suitable assumptions on operators and the form of invariant sets. We find a class of positive solutions having the selfsimilar description.

Finally, we investigate the convergence of solutions for a second-order closure model to the obtained selfsimilar solution.

\section{Modelling the turbulent transport in a shearless mixing layer}

The subject of the study is the problem of interaction and mixing between two semiinfinite homogeneous turbulent flow fields of different scales. As the flow evolves these two different turbulent fields penetrate and diffuse into one another. To describe correctly the physical model, it is proposed to consider the hypothesis of equality to zero of the fifthorder cumulants under the condition of nonzero fourth-order cumulants. The starting point is the following system of average equations:

$$
\begin{gathered}
\frac{\partial e_{h}}{\partial t}=-\frac{\partial\left\langle e_{h} w\right\rangle}{\partial z}-\frac{c_{1}}{\tau}\left[e_{h}-\frac{2}{3} E\right]-\frac{2}{3} \epsilon, \\
\frac{\partial \epsilon}{\partial t}=\frac{\partial}{\partial z}\left[c_{d} \tau\left\langle w^{2}\right\rangle \frac{\partial \epsilon}{\partial z}\right]+\frac{c_{\epsilon_{1}}}{\tau} \beta g\langle w \theta\rangle-c_{\epsilon_{2}} \frac{\epsilon}{\tau} \\
\frac{\partial\left\langle w^{2}\right\rangle}{\partial t}=-\frac{\partial\left\langle w^{3}\right\rangle}{\partial z}+2 \beta g\langle w \theta\rangle-\frac{c_{1}}{\tau}\left[\left\langle w^{2}\right\rangle-\frac{2}{3} E\right]-\frac{2}{3} \epsilon .
\end{gathered}
$$

In the absence of mean shear velocity the horizontal component $e_{h}$ of the turbulent kinetic energy takes the following form:

$$
e_{h}=\left(\left\langle u^{2}\right\rangle+\left\langle v^{2}\right\rangle\right) / 2
$$

As usual, the sign $\langle\cdot\rangle$ denotes average values, $\left\langle u^{2}\right\rangle,\left\langle v^{2}\right\rangle,\left\langle w^{2}\right\rangle$ are the one-point velocity correlation of the second-order, $\tau=E / \epsilon$ is the time scale of turbulence. Here $E, \epsilon$ are the kinetic energy and spectral flux of the turbulent kinetic energy respectively. The volumetric expansion coefficient is $\beta=1 / \Theta$, where $\Theta$ and $\theta$ are the mean and variance potential temperature respectively. The constants involved in the model with the lower 
case letters are denoted by $c_{* *}$. We complete the system by the transport equation for the triple correlation of the vertical velocity fluctuation:

$$
\frac{\partial\left\langle w^{3}\right\rangle}{\partial t}=-\frac{\partial C}{\partial z}-3\left\langle w^{2}\right\rangle \frac{\partial\left\langle w^{2}\right\rangle}{\partial z}+3 \beta g\left\langle w^{2} \theta\right\rangle-c_{2} \frac{\left\langle w^{3}\right\rangle}{\tau}
$$

Algebraic parametrizations for the fourth-order cumulant $C$, the triple correlation $\left\langle w^{2} \theta\right\rangle$ and the vertical heat flux $\langle w \theta\rangle$ can be written as [4]:

$$
\begin{gathered}
C=-\frac{\tau}{c_{3}}\left[6\left\langle w^{3}\right\rangle \frac{\partial\left\langle w^{2}\right\rangle}{\partial z}+4\left\langle w^{2}\right\rangle \frac{\partial\left\langle w^{3}\right\rangle}{\partial z}\right] \\
\left\langle w^{2} \theta\right\rangle=-\frac{\tau}{c_{4}}\left[\left\langle w^{3}\right\rangle \frac{\partial \Theta}{\partial z}-2 \beta g\left\langle w \theta^{2}\right\rangle\right], \quad\left\langle w \theta^{2}\right\rangle=-\frac{\tau}{c_{5}}\left\langle w^{2}\right\rangle \frac{\partial\left\langle\theta^{2}\right\rangle}{\partial z}, \\
\langle w \theta\rangle=-\frac{\tau}{c_{\theta_{1}}}\left\langle w^{2}\right\rangle \frac{\partial \Theta}{\partial z} \equiv-\frac{\tau N^{2}}{\beta g c_{\theta_{1}}}\left\langle w^{2}\right\rangle, \quad N^{2}=\beta g \frac{\partial \Theta}{\partial z} \\
\left\langle\theta^{2}\right\rangle=-\frac{\tau}{c_{r}}\langle w \theta\rangle \frac{\partial \Theta}{\partial z}=-\frac{\tau N^{2}}{\beta g r}\langle w \theta\rangle=\left(\frac{\tau N^{2}}{\beta g}\right)^{2} \frac{\left\langle w^{2}\right\rangle}{c_{\theta_{1}} r}
\end{gathered}
$$

where $r=\tau / \tau_{\theta}, \tau_{\theta}$ is the time scale of potential temperature variance, $N$ is the BruntVaisala frequency. On using the balance approximation between exchange mechanism and dissipation, the equation for the horizontal component $e_{h}$ of the turbulent kinetic energy is simplified and has the following form:

$$
-c_{1}\left[e_{h}-\frac{2}{3}\left(e_{h}+\frac{\left\langle w^{2}\right\rangle}{2}\right)\right]=\frac{2}{3}\left(e_{h}+\frac{\left\langle w^{2}\right\rangle}{2}\right) .
$$

Hence,

$$
e_{h}=\frac{c_{1}-1}{c_{1}+2}\left\langle w^{2}\right\rangle, \quad E=\frac{31}{2\left(c_{1}+2\right)}\left\langle w^{2}\right\rangle, \quad \tau=\frac{3 c_{1}}{2\left(c_{1}+2\right)} \frac{\left\langle w^{2}\right\rangle}{\epsilon} .
$$

By the obtained relations, in the case of absence stratification i.e. $N \equiv 0$ the equation can be rewritten as:

$$
\begin{gathered}
\frac{\partial\left\langle w^{2}\right\rangle}{\partial t}=-\frac{\partial\left\langle w^{3}\right\rangle}{\partial z}-\frac{c_{1}}{c_{1}+2} \frac{\left\langle w^{2}\right\rangle}{\tau} \\
\frac{\partial \epsilon}{\partial t}=\frac{\partial}{\partial z}\left[c_{d} \tau\left\langle w^{2}\right\rangle \frac{\partial \epsilon}{\partial z}\right]-c_{\epsilon_{2}} \frac{\epsilon}{\tau} \\
\frac{\partial\left\langle w^{3}\right\rangle}{\partial t}=\frac{\partial}{\partial z}\left[\frac{\tau}{c_{3}}\left(6\left\langle w^{3}\right\rangle \frac{\partial\left\langle w^{2}\right\rangle}{\partial z}+4\left\langle w^{2}\right\rangle \frac{\left\langle\partial w^{3}\right\rangle}{\partial z}\right)\right]-3\left\langle w^{2}\right\rangle \frac{\partial\left\langle w^{2}\right\rangle}{\partial z}-c_{2} \frac{\left\langle w^{3}\right\rangle}{\tau} .
\end{gathered}
$$

It follows from the formula for $C$ [4] that the contribution of the second term in the algebraic model for the cumulant $C$ is essential. Thus the governing equations are:

$$
\frac{\partial\left\langle w^{2}\right\rangle}{\partial t}=-\frac{\partial\left\langle w^{3}\right\rangle}{\partial z}-\alpha \frac{\left\langle w^{2}\right\rangle}{\hat{\tau}}
$$




$$
\begin{gathered}
\frac{\partial\left\langle w^{3}\right\rangle}{\partial t}=\frac{\partial}{\partial z}\left[\kappa \hat{\tau}\left\langle w^{2}\right\rangle \frac{\partial\left\langle w^{3}\right\rangle}{\partial z}\right]-3\left\langle w^{2}\right\rangle \frac{\partial\left\langle w^{2}\right\rangle}{\partial z}-\gamma \frac{\left\langle w^{3}\right\rangle}{\hat{\tau}}, \\
\frac{\partial \epsilon}{\partial t}=\frac{\partial}{\partial z}\left[\delta \hat{\tau}\left\langle w^{2}\right\rangle \frac{\partial \epsilon}{\partial z}\right]-\varrho \frac{\epsilon}{\hat{\tau}}
\end{gathered}
$$

where $\alpha=2 / 3, \kappa=6 c_{1} / c_{3}\left(c_{1}+2\right), \gamma=2 c_{2}\left(c_{1}+1\right) / 3 c_{1}, \delta=3 c_{1} c_{d} / 2\left(c_{1}+2\right), \varrho=$ $2 c_{\epsilon_{2}}\left(c_{1}+2\right) / 3 c_{1}, \hat{\tau}=\left\langle w^{2}\right\rangle / \epsilon$.

\section{Invariant sets}

We briefly recall the special terminology of Symmetry Analysis. For the detailed description of extra materials see, for example [9], [10].

Consider the system of evolution equations $\mathcal{F}$ :

$$
u_{t}^{i}=\mathcal{F}^{i}\left(t, x_{1}, \ldots, x_{n}, u^{1}, \ldots, u_{\lambda}^{k}, \ldots,\right)
$$

where $i=1, \ldots, m, u_{\lambda}^{k}=\partial^{\lambda} u^{k} / \partial x_{1}^{\lambda_{1}} \ldots \partial x_{n}^{\lambda_{n}}$.

A set(manifold) $\mathcal{H}$ given by equations:

$$
h_{i}\left(t, x_{1}, \ldots, x_{n}, \ldots, u^{1}, \ldots, u^{m}, \ldots, u_{\lambda}^{k}, \ldots\right)=0
$$

is said to be the invariant set (manifold) of the system $\mathcal{F}$ if

$$
\begin{gathered}
\left.V_{\mathcal{F}}\left(h^{i}\right)\right|_{[\mathcal{H}]_{0}}=0 \\
V_{\mathcal{F}}=\frac{\partial}{\partial t}+\sum_{i=1}^{m} \mathcal{F}^{i} \frac{\partial}{\partial u^{1}}+\sum_{i=1}^{m} D^{\alpha}\left(\mathcal{F}^{i}\right) \frac{\partial}{\partial u_{\alpha}^{i}}
\end{gathered}
$$

where $\alpha=\left(\alpha_{1}, \ldots, \alpha_{n}\right), D^{\alpha}=D_{x_{1}}^{\alpha_{1}} \ldots D_{x_{n}}^{\alpha_{n}}$. Here $[\mathcal{H}]_{0}$ denotes the equations $\mathcal{H}$ and its differential prolongations with respect to $x_{1}, \ldots, x_{n}$.

The invariant condition can be written in the following equivalent form:

$$
\left.\left.D_{t}\left(h_{i}\right)\right|_{[\mathcal{F}]_{0}}\right|_{[\mathcal{H}]_{0}}=0 .
$$

In an application of invariant relations between the system $\mathcal{F}$ and set (manifold) $\mathcal{H}$ we demonstrate the following useful

Theorem 3.1 ([10]) Assume that the system $\mathcal{F}$ has an invariant set(manifold) of the form $\mathcal{H}$ which is solved with respect to higher derivatives and the initial conditions are given by $u_{n_{i}}^{i}\left(x_{0}, t_{0}\right)=c_{i k_{i}}, c_{i k_{i}} \in R$, then in some neighborhood of $\left(x_{0}, t_{0}\right) \in R^{2}$ there exists a unique smooth solution of $\mathcal{F}$.

As the first result that uses the above notion we prove that the set

$$
D=\left\{\left\langle w^{2}\right\rangle,\left\langle w^{3}\right\rangle, \hat{\tau}: \mathcal{H}^{1}\left(\left\langle w^{2}\right\rangle,\left\langle w^{3}\right\rangle, \hat{\tau}\right) \equiv\left\langle w^{3}\right\rangle+\delta \hat{\tau}\left\langle w^{2}\right\rangle\left\langle w^{2}\right\rangle_{z}=0\right\}
$$

is invariant under the flow generated by equation (2.2). 
Theorem 3.2 Let the triple $\left\langle w^{2}\right\rangle,\left\langle w^{3}\right\rangle$, $\epsilon$ be a sufficiently smooth solution of (2.1)-(2.3). Assume also that:

$$
\frac{\partial \hat{\tau}}{\partial z}=0, \quad \frac{\partial \hat{\tau}}{\partial t}=2 \alpha-\gamma+\frac{3}{\delta}, \quad \kappa=\delta
$$

Then:

(i) the equation (2.2) admits the invariant set D;

(ii) the system (2.1)-(2.3) on the invariant set D is equivalent to:

$$
\begin{gathered}
\left\langle w^{2}\right\rangle=2 \alpha \epsilon-\gamma \epsilon+\frac{3 \epsilon}{\delta}, \\
\left\langle w^{3}\right\rangle=-\delta \hat{\tau}\left\langle w^{2}\right\rangle \frac{\partial\left\langle w^{2}\right\rangle}{\partial z}, \\
\frac{\partial \epsilon}{\partial t}=\frac{\partial}{\partial z}\left[\delta \hat{\tau}^{2} \epsilon \frac{\partial \epsilon}{\partial z}\right]-\varrho \frac{\epsilon}{\hat{\tau}} .
\end{gathered}
$$

Proof. (i) Calculating the time derivative $D_{t} \mathcal{H}$ we obtain:

$$
D_{t} \mathcal{H}^{1}=\frac{\partial\left\langle w^{3}\right\rangle}{\partial t}+\delta \frac{\partial \hat{\tau}}{\partial t} \frac{\partial\left\langle w^{2}\right\rangle}{\partial z}\left\langle w^{2}\right\rangle+\delta \hat{\tau} \frac{\partial\left\langle w^{2}\right\rangle}{\partial t} \frac{\partial\left\langle w^{2}\right\rangle}{\partial z}+\delta \hat{\tau}\left\langle w^{2}\right\rangle \frac{\partial^{2}\left\langle w^{2}\right\rangle}{\partial t \partial z} .
$$

Using the equation (2.2) we can rewrite (3.6) as

$$
\begin{aligned}
& D_{t} \mathcal{H}^{1}=\frac{\partial}{\partial z}\left[\kappa \hat{\tau}\left\langle w^{2}\right\rangle \frac{\partial\left\langle w^{3}\right\rangle}{\partial z}\right]-3\left\langle w^{2}\right\rangle \frac{\partial\left\langle w^{2}\right\rangle}{\partial z}-\gamma \frac{\left\langle w^{3}\right\rangle}{\hat{\tau}}+ \\
& +\delta \frac{\partial \hat{\tau}}{\partial t} \frac{\partial\left\langle w^{2}\right\rangle}{\partial z}\left\langle w^{2}\right\rangle+\delta \hat{\tau} \frac{\partial\left\langle w^{2}\right\rangle}{\partial t} \frac{\partial\left\langle w^{2}\right\rangle}{\partial z}+\delta \hat{\tau}\left\langle w^{2}\right\rangle \frac{\partial^{2}\left\langle w^{2}\right\rangle}{\partial t \partial z} .
\end{aligned}
$$

Replacing the derivative $\partial\left\langle w^{3}\right\rangle / \partial z$ into the diffusion operator in (2.2) by its representation

$$
\frac{\partial\left\langle w^{3}\right\rangle}{\partial z}=-\frac{\partial\left\langle w^{2}\right\rangle}{\partial t}-\alpha \frac{\left\langle w^{2}\right\rangle}{\hat{\tau}}
$$

from equation (2.1) we obtain

$$
\begin{gathered}
D_{t} \mathcal{H}^{1}=-\kappa \frac{\partial \hat{\tau}}{\partial z} \frac{\partial\left\langle w^{2}\right\rangle}{\partial t}\left\langle w^{2}\right\rangle-(\kappa-\delta) \hat{\tau} \frac{\partial\left\langle w^{2}\right\rangle}{\partial t} \frac{\partial\left\langle w^{2}\right\rangle}{\partial z}- \\
-(\kappa-\delta) \hat{\tau}\left\langle w^{2}\right\rangle \frac{\partial^{2}\left\langle w^{2}\right\rangle}{\partial z \partial t}-\left(2 \alpha \kappa-\frac{\partial \hat{\tau}}{\partial t} \delta-\gamma \delta+3\right)\left\langle w^{2}\right\rangle \frac{\partial\left\langle w^{2}\right\rangle}{\partial z} .
\end{gathered}
$$

It follows from the hypothesis (3.2) that $\left.\left.D_{t} \mathcal{H}^{1}\right|_{[\mathcal{F}]_{0}}\right|_{[\mathcal{H}]_{0}}=0$.

(ii) Indeed, the proof of this assertion is the consequence of (i). We only note that (2.2) is fulfilled for the functions which belong to the set $D$, and substituting

$$
\epsilon_{a}=\frac{\left\langle w_{a}^{2}\right\rangle}{(\varrho-\alpha)\left(t+t_{0}\right)}
$$

into (2.3) yields equation (2.1). This completes the proof of theorem.

Theorem 3.2 is of special interest in view of its application to Turbulent Models. 
Corollary 3.1 The equation $\mathcal{H}^{1}\left(\left\langle w^{2}\right\rangle,\left\langle w^{3}\right\rangle, \hat{\tau}\right)=0$ that defines an invariant set of (2.1)(2.3) coincides with the algebraic triple correlation model or the tensor-invariant model [8].

In other words, the algebraic expression represents the equation of an invariant set (manifold) generated by the differential equation for the triple correlation.

Corollary 3.2 There exists no differential constraints of the form

$$
\mathcal{H}^{n} \equiv\left\langle w^{3}\right\rangle-H^{n}\left(\left\langle w^{2}\right\rangle,\left\langle w^{3}\right\rangle,\left\langle w^{3}\right\rangle_{1}, \ldots\left\langle w^{3}\right\rangle_{n}, \hat{\tau}\right)=0
$$

for $n>1$ where $\left\langle w^{3}\right\rangle_{n}$ denotes the $n$-order derivative, $H^{n}$ is a sufficiently smooth function.

Proof. This follows by substituting $\left\langle w^{3}\right\rangle=H^{(n)}\left(\left\langle w^{2}\right\rangle,\left\langle w^{3}\right\rangle,\left\langle w^{3}\right\rangle_{1}, \ldots\left\langle w^{3}\right\rangle_{n}, \hat{\tau}\right)$ into equations (2.1), (2.2) and comparing the orders of higher derivatives. This yields $2 n+1=n+2$ that is $n=1$.

\section{Selfsimilar solutions}

Theorem 3.2 enables us to reduce (2.1)-(2.3) to the algebraic differential expressions (3.3)(3.5) which can be easier analyzed. Using the obtained reduction we give the selfsimilar description for (2.1)-(2.3). It should be recalled that the solution of the equation (or the system of equations) is said to be selfsimilar for the equation if this solution is invariant under the parametric group of scale transformation.

Appropriate selfsimilar solution to our problem is a solution of the following form:

$$
\begin{gathered}
\epsilon_{a}=\frac{h(\xi)}{\left.t+t_{0}\right)^{3 \mu+\nu}}, \quad\left\langle w_{a}^{2}\right\rangle=\frac{f(\xi)}{\left(t+t_{0}\right)^{2 \mu}}, \quad\left\langle w_{a}^{3}\right\rangle=\frac{q(\xi)}{\left(t+t_{0}\right)^{3 \mu}}, \\
\xi=\frac{z-z_{0}}{L}, \quad L=\lambda\left(t+t_{0}\right)^{\nu}, \quad z_{0}=\lambda_{0} L+\lambda_{1}, \quad t_{0}>0,
\end{gathered}
$$

where $\lambda, \lambda_{i}$ are model constants and $t_{0}$ is a parameter. A straightforward calculation yields that if we choose $\nu=1-\mu$, then the original system is transformed to the system of ordinary differential equations for the profiles $f, q$ and $h$ :

$$
\begin{gathered}
2 \mu f+(1-\mu)\left(\xi+\lambda_{0}\right) f_{\xi}-g_{\xi}-\alpha h=0, \\
\kappa\left(\frac{f^{2}}{h} q_{\xi}\right)_{\xi}-3 f f_{\xi}-\left(\xi+\lambda_{0}\right) q_{\xi}-\gamma \frac{q h}{f}+3 \mu q=0, \\
\delta\left(\frac{f^{2}}{h} h_{\xi}\right)_{\xi}-\left(\xi+\lambda_{0}\right) h_{\xi}+(2 \mu+1) h-\varrho \frac{h^{2}}{f}=0 .
\end{gathered}
$$

The free similarity exponent $\mu$ has to be determined from a solution of the obtained nonlinear eigenvalue problem. This is a typical situation appearing in nonlinear diffusion problems where a conservation law does not exist. The boundary conditions are determined by the physical model: the functions $f(\xi), q(\xi), h(\xi)$ tend to the positive limits as 
$\xi$ tends to $\pm \infty$. The analysis of behavior of the integral curves to the eigenvalue problem encounters certain obstacles. In the case of a simpler $K-\varepsilon$ model this analysis was partially formed in [11].

To solve the problem we use the existence of the invariant set obtained in Theorem 3.2. Let us check the conditions of the theorem. For this purpose, we consider the equation for $\hat{\tau}$. On calculating the time derivative $\hat{\tau}_{t}$ we obtain:

$$
\begin{gathered}
\frac{\partial \hat{\tau}}{\partial t}=\frac{1}{\epsilon} \frac{\partial\left\langle w^{2}\right\rangle}{\partial t}-\frac{\left\langle w^{2}\right\rangle}{\epsilon^{2}} \frac{\partial \epsilon}{\partial t}= \\
-\frac{1}{\epsilon} \frac{\partial\left\langle w^{3}\right\rangle}{\partial z}-\alpha-\delta \hat{\tau}^{2} \frac{\partial^{2}\left\langle w^{2}\right\rangle}{\partial z^{2}}+\delta\left\langle w^{2}\right\rangle \hat{\tau} \frac{\partial^{2} \hat{\tau}}{\partial z^{2}}-\delta \frac{\hat{\tau}^{2}}{\left\langle w^{2}\right\rangle}\left(\frac{\partial\left\langle w^{2}\right\rangle}{\partial z}\right)^{2}+\varrho .
\end{gathered}
$$

Thus the equation for $\hat{\tau}$ has the form:

$$
\frac{\partial \hat{\tau}}{\partial t}=\delta\left\langle w^{2}\right\rangle \hat{\tau} \frac{\partial^{2} \hat{\tau}}{\partial z^{2}}-\frac{\hat{\tau}}{\left\langle w^{2}\right\rangle}\left[\frac{\partial\left\langle w^{3}\right\rangle}{\partial z}+\delta \hat{\tau}\left\langle w^{2}\right\rangle \frac{\partial^{2}\left\langle w^{2}\right\rangle}{\partial z^{2}}+\delta \hat{\tau}\left(\frac{\partial\left\langle w^{2}\right\rangle}{\partial z}\right)^{2}\right]-\alpha+\varrho .
$$

Obviously, it is sufficient to check the conditions of the theorem only for the functions $\left(\left\langle w^{2}\right\rangle,\left\langle w^{3}\right\rangle, \hat{\tau}\right) \in D$. It is clear that $\hat{\tau}=(\varrho-\alpha)\left(t+t_{0}\right)$ is a solution of (4.5). Setting

$$
\varrho-\alpha=(2 \alpha \delta-\gamma \delta+3) / \delta
$$

and applying Theorem 3.2 we obtain that system (4.2)-(4.4)) admits a reduction. As a result, we have the following boundary value problem for $h(\xi)$ :

$$
\begin{gathered}
\delta\left(w_{c}^{2} h h_{\xi}\right)_{\xi}+(1-\mu)\left(\xi+\lambda_{0}\right) h_{\xi}=0, \quad w_{c}=\varrho-\alpha, \quad \mu=\frac{\alpha}{2(\varrho-\alpha)}, \\
h(-\infty)=a_{-} t_{0}^{2 \mu+1}, \quad h(+\infty)=a_{+} t_{0}^{2 \mu+1}
\end{gathered}
$$

where $a_{ \pm}=\lim _{z \rightarrow \pm \infty} \epsilon(z, 0), a_{ \pm}>0$. Taking into account (4.6) the following relations between the model constants hold:

$$
\delta=\frac{3}{\varrho-\gamma}, \quad \gamma=\frac{3}{2} \alpha, \quad \varrho-\gamma>0
$$

We prove the existence and uniqueness theorem for the problem (4.7), (4.8), present in detailed the qualitative properties of the solution. Note that this solution is essentially different from the well-known Barenblatt's solutions [12]; first, a class of positive solution to the porous medium equation was introduced into consideration in [13].

It will be convenient to deal with the function $U(\bar{\xi})=h^{2}\left(\bar{\xi}-\lambda_{0}\right)$, where $\bar{\xi}=\xi+\lambda_{0}$. Then (4.7), (4.8) is rewritten as

$$
\begin{gathered}
\delta(\rho-\alpha)^{2} U_{\bar{\xi} \bar{\xi}}+(1-\mu) \bar{\xi} U^{-1 / 2} U_{\bar{\xi}}=0 \\
U(-\infty)=\left(a_{-} t_{0}^{2 \mu+1}\right)^{2}, \quad U(+\infty)=\left(a_{+} t_{0}^{2 \mu+1}\right)^{2} .
\end{gathered}
$$


To find a solution to (4.10), (4.11) we consider a family of the Cauchy problem to the equation $(4.10)$ on $(0,+\infty)$ with the initial data:

$$
U(0)=\eta, \quad U_{\bar{\xi}}(0)=\varphi
$$

where $\eta, \varphi$ are positive numbers. By ODE Theory, there exists a unique local solution $\widehat{U}$ for every $\eta, \varphi$.

Lemma 4.1 The solution $\widehat{U}$ of the Cauchy problem (4.10), (4.12) is increasing and concave positive function on an interval where the function $\widehat{U}$ exists.

Proof. Positiveness of the derivative $\widehat{U}_{\bar{\xi}}$ is deduced from the following general property that holds for $P=\epsilon_{\bar{\xi}}$ : the order of zero of the function $P$ in the domain $\{\epsilon>0\}$ for $t>0$ counted with multiplicity is finite with the exception of the case $\epsilon \equiv$ const.

Let us suppose that $\widehat{U}_{\bar{\xi}}$ vanishes at a point $\bar{\xi}_{0}$. By differentiating of equation (4.10) we can conclude that all derivative of $\widehat{U}$ vanishes at the same point $\bar{\xi}_{0}$. This implies that $P$ has a zero of infinite order which contradicts the above presented property. Thus $\widehat{U}_{\bar{\xi}}$ cannot be zero for $\bar{\xi}>0$.

Corollary 4.1 The solution $\widehat{U}$ of (4.10), (4.12) exists for all $\bar{\xi} \geq 0$.

Proof. This directly follows from the inequalities $\widehat{U}_{\bar{\xi}}>0, \widehat{U}_{\bar{\xi} \bar{\xi}}<0$.

Lemma $4.2 \widehat{U}_{\bar{\xi}} \rightarrow 0$ as $\bar{\xi} \rightarrow \infty$.

Proof. From equation (4.10) we find

$$
\widehat{U}_{\bar{\xi}}=\varphi \exp \left(-\frac{(1-\mu)}{\delta(\rho-\alpha)^{2}} \int_{0}^{\bar{\xi}} s \widehat{U}^{-1 / 2} d s\right) .
$$

To estimate $\widehat{U}_{\bar{\xi}}$ we use the inequality $\widehat{U} \leq \eta+\varphi \bar{\xi}$ (see, Corollary4.1). It is not hard to see that

$$
\widehat{U}_{\bar{\xi}} \approx \varphi \exp \left(-\frac{2(1-\mu)}{3 \delta(\rho-\alpha)^{2}} \varphi^{-1 / 2} \bar{\xi}^{3 / 2}\right)
$$

for sufficiently large values of $\bar{\xi}$.

Corollary 4.2 The function $\widehat{U}$ has a horizontal asymptote. Moreover, the following estimates hold

$$
\varphi^{5 / 4} \frac{\sqrt{3 \delta}(\rho-\alpha)}{2 \sqrt{2} \sqrt{1-\mu}} \sqrt{\pi}+\eta \leq \lim _{\bar{\xi} \rightarrow \infty} \widehat{U}(\bar{\xi}) \leq \varphi^{3 / 2} \frac{3 \delta(\rho-\alpha)^{2}}{2(1-\mu)} e+\eta .
$$

The proof of the left-hand side of inequality follows from $\int_{-\infty}^{\infty} \exp \left(-\bar{\xi}^{2}\right) d \bar{\xi}=\sqrt{\pi}$.

Remark 4.1 Setting $\varphi=0$ in (4.12), we have $\widehat{U} \equiv \eta$ for all $\bar{\xi} \geq 0$. 
Along with (4.10), (4.12), we consider the problem with the initial data

$$
\widetilde{U}(0)=\eta, \quad \widetilde{U}(0)_{\bar{\xi}}=-\varphi .
$$

A similar analysis as in Lemma 4.1 enables us to establish

Lemma 4.3 The solution $\widetilde{U}$ of (4.10), (4.13) is a decreasing convex function on an interval where $\widetilde{U}>0$.

The next Lemma guarantees positiveness of $\widetilde{U}$ and therefore, solvability of (4.10), (4.13) in a class of positive functions defined on $[0, \infty)$.

Lemma 4.4 If

$$
f_{a}(0) \leq \widetilde{U}(0), \quad\left|\widetilde{U}_{\bar{\xi}}\right|<\left|f_{a}(0)_{\bar{\xi}}\right|
$$

then $\widetilde{U}>0$ on $[0, \infty)$.

Here the function $f_{a}(\bar{\xi})$ denotes Barenblatt's selfsimilar solution [12] Proof of Lemma 4.4 is based on analysis of behavior of the integral curves to (4.10). Applying the comparison theorem to the functions $\widetilde{U}$ and $f_{a}$ (taking into account (4.14)) we obtain that $\widetilde{U}$ belongs to a class of integral curves that do not intersect $\bar{\xi}$-axis and have horizontal asymptotes depending continuously on the values of derivative $\widetilde{U}_{\bar{\xi}}$ at zero. Moreover, $\bar{\xi}$-axis is not asymptote for the integral curves.

Combining functions $\widehat{U}$ and $\widetilde{U}$ we find the solution of the equation $(4.10)$ on $(-\infty,+\infty)$. Indeed, this equation is invariant under the transformation $\bar{\xi} \rightarrow-\bar{\xi}$. Therefore, the function $U(\bar{\xi})$ equals $\widehat{U}\left(\bar{\xi}_{+}\right)$for $\bar{\xi} \geq 0$ and $\widetilde{U}\left(-\bar{\xi}_{+}\right)$for $\bar{\xi}<0$ is a solution of $(4.10)$ on $(-\infty, \infty)$ (where $\bar{\xi}_{+}=\{\bar{\xi} \geq 0\}$ ). It then follows from the properties of $\bar{U}$ and $\widetilde{U}$ that for $U$ holds

Lemma 4.5 The function $U$ is increasing over $(-\infty,+\infty)$ and has finite limits as $\bar{\xi} \rightarrow$ $\pm \infty$. Moreover, $U$ has convex and concave profiles for $\bar{\xi}<0$ and $\bar{\xi}>0$, respectively. The derivative $U_{\bar{\xi}}$ satisfies $0<U_{\bar{\xi}} \leq U_{\bar{\xi}}(0)<\left|f_{a \bar{\xi}}(0)\right|$.

Using the previous Lemmas we arrive at the following

Theorem 4.1 For any positive finite numbers $a_{-}$and $a_{+}\left(a_{-} \leq a_{+}\right)$there exists a parameter $t_{0}$ such that the boundary value problem (4.10), (4.12) (respectively (4.7), (4.8)) has a unique positive solution.

Remark 4.2 The test configurations of profiles of the spectral flux $\epsilon$ obtained by numerical and experimental methods (see, for example [14], [15]) coincides qualitatively with profiles of $\epsilon_{a}$.

Once we have determined $h(\xi)$, we can find $\epsilon_{a}$ by the formula $\epsilon_{a}(z, t)=h(\xi) /\left(t+t_{0}\right)^{2 \mu+1}$, $\left\langle w_{a}^{2}\right\rangle$ is defined from the relation $\left\langle w_{a}^{2}\right\rangle=\hat{\tau} \epsilon_{a}=(\rho-\alpha)\left(t+t_{0}\right) \epsilon_{a}$. For the triple correlation $\left\langle w_{a}^{3}\right\rangle$ the following holds

$$
\left\langle w_{a}^{3}\right\rangle=-\delta(\rho-\alpha)\left(t+t_{0}\right)\left\langle w_{a}^{2}\right\rangle \frac{\partial\left\langle w_{a}^{2}\right\rangle}{\partial \xi}
$$

Thus we have proved 
Theorem 4.2 Let $\kappa=\delta, \delta=3 /(\rho-\gamma)$ and $\gamma=(3 / 2) \alpha$, assume that $\rho-\gamma>0$. Then there exists a solution $\left\langle w_{a}^{2}\right\rangle,\left\langle w_{a}^{3}\right\rangle, \epsilon_{a}$ of system (2.1)-(2.3) which represents the selfsimilar description of the shearless turbulence mixing layer.

\section{Convergence to the selfsimilar solution}

In this section we compare asymptotic behavior of a solution to a turbulent diffusion model of gradient type ("standard" second-order model of turbulence) with the selfsimilar solution $\left\langle w_{a}^{2}\right\rangle,\left\langle w_{a}^{3}\right\rangle, \epsilon_{a}$ obtained in Section 4 .

This model consists of a system of two reaction-diffusion partial differential equations, namely:

$$
\begin{gathered}
\frac{\partial\left\langle w^{2}\right\rangle}{\partial t}=\frac{\partial}{\partial z}\left[\delta \hat{\tau}\left\langle w^{2}\right\rangle \frac{\partial\left\langle w^{2}\right\rangle}{\partial z}\right]-\alpha \frac{\left\langle w^{2}\right\rangle}{\hat{\tau}}, \\
\frac{\partial \epsilon}{\partial t}=\frac{\partial}{\partial z}\left[\delta \hat{\tau}\left\langle w^{2}\right\rangle \frac{\partial \epsilon}{\partial z}\right]-\varrho \frac{\epsilon}{\hat{\tau}}
\end{gathered}
$$

with initial data

$$
\left\langle w^{2}(z, 0)\right\rangle=\left\langle w_{0}^{2}(z)\right\rangle, \quad \epsilon(z, 0)=\epsilon_{0}(z),
$$

where $\left\langle w_{0}^{2}(z)\right\rangle, \epsilon(z, 0)=\epsilon_{0}(z)$ are given bounded, positive and continuous function on $R$ with finite $\lim _{t \rightarrow \pm \infty}\left\langle w_{0}^{2}(z)\right\rangle=b_{ \pm}, \lim _{t \rightarrow \pm \infty} \epsilon_{0}(z)=a_{ \pm}$. Here $a_{ \pm}, b_{ \pm}$are positive numbers. We show that the large time behavior of the solution for (5.1)-(5.3) can be well approximated by $\left(\left\langle w_{a}^{2}\right\rangle, \epsilon_{a}\right)$.

First, we prove the existence result to the Cauchy problem for the model using an approach suggested in [16]. To achieve the existence of a solution to (5.1)-(5.3), we begin with constructing approximate solutions $\left\langle w_{k}^{2}\right\rangle, \epsilon_{k}$ for $k=1, \ldots$, as a solution to the system:

$$
\begin{aligned}
\frac{\partial\left\langle w_{k}^{2}\right\rangle}{\partial t} & =\frac{\partial}{\partial z}\left[\delta \psi\left(\hat{\tau}_{k}\left(z, t-k^{-1}\right)\right)\left\langle w_{k}^{2}\left(z, t-k^{-1}\right)\right\rangle \frac{\partial\left\langle w_{k}^{2}\right\rangle}{\partial z}\right]-\alpha \frac{\left\langle w_{k}^{2}\right\rangle}{\psi\left(\hat{\tau}_{k}\left(z, t-k^{-1}\right)\right)}, \\
\frac{\partial \epsilon_{k}}{\partial t} & =\frac{\partial}{\partial z}\left[\delta \psi\left(\hat{\tau}_{k}\left(z, t-k^{-1}\right)\left\langle w_{k}^{2}\left(z, t-k^{-1}\right)\right)\right\rangle \frac{\partial \epsilon_{k}}{\partial z}\right]-\varrho \frac{\epsilon_{k}}{\psi\left(\hat{\tau}_{k}\left(z, t-k^{-1}\right)\right)}
\end{aligned}
$$

in $Q_{k}=[-k, k] \times[0, k]$ under the conditions:

$$
\begin{array}{cc}
\frac{\partial\left\langle w_{k}^{2}( \pm k, t)\right\rangle}{\partial t}=0, & \frac{\partial \epsilon( \pm k, t)}{\partial t}=0, \\
\left\langle w_{k}^{2}(z, 0)\right\rangle=\left\langle w_{0 k}^{2}(z)\right\rangle, & \epsilon_{k}(z, 0)=\epsilon_{0 k}(z),
\end{array}
$$

where

$$
\left\langle w_{0 k}^{2}\right\rangle, \epsilon_{0 k} \in C^{\infty}([-k, k]), \quad \frac{\partial\left\langle w_{0 k}^{2}( \pm k)\right\rangle}{\partial z}=0, \quad \frac{\partial \epsilon_{0 k}( \pm k)}{\partial z}=0,
$$

and the sequences positive bounded functions $\left\langle w_{0 k}^{2}\right\rangle, \epsilon_{0 k}$ convergence to $\left\langle w_{0}^{2}\right\rangle, \epsilon_{0}$ in $C_{l o c}(R)$ as $k$ tends to infinity. The function $\psi(s), s \in R^{+}$is defined as follows:

$$
\psi \in C^{\infty}\left(R^{+}\right), \quad \psi(s)=s \quad \text { for } \quad s \in[d, \infty), \quad \text { where } \quad d=\min _{z \in R} \hat{\tau}(z, 0),
$$




$$
\psi(s)=d / 2 \quad \text { for } \quad s \in[0, d / 2], \quad \psi^{\prime}(s)>0, \quad \text { for } \quad s \in(d / 2, d],
$$

and

$$
\left\langle w_{k}^{2}(z, t)\right\rangle=\left\langle w_{0 k}^{2}(z)\right\rangle, \quad \epsilon_{k}(z, t)=\epsilon_{0 k}(z), \quad \text { for } \quad-k^{-1} \leq t \leq 0 .
$$

For each fixed $k=1,2, \ldots$, a solution to problem (5.4)-(5.7) in $Q_{k}$ is constructed successively in the layers

$$
Q_{k}^{j}=[-k, k] \times\left[(j-1) k^{-1}, j k^{-1}\right], \quad j=1, \ldots, k^{2},
$$

of the cylinder $Q_{k}^{j}$. The existence of a unique solution of class $C^{2+\alpha, 1+\alpha / 2}$ in each layer ensues from [17]. According to the maximum principle, the functions $\left\langle w_{k}^{2}\right\rangle, \epsilon_{k}$ are upper bounded uniformly in $k$. The a priori lower bounds for $\left\langle w_{k}^{2}\right\rangle, \epsilon_{k}$ in $Q_{k}$ follows from comparison with a spatially homogeneous solution to problem (5.4)-(5.7), and we have:

$$
\begin{gathered}
\min _{z \in[-k, k]}\left\langle w_{0 k}^{2}(z)\right\rangle\left[\frac{\min _{z \in[-k, k]} \hat{\tau}(z, 0)}{\min _{z \in[-k, k]} \hat{\tau}(z, 0)+(\varrho-\alpha) k}\right]^{\alpha} \leq\left\langle w_{k}^{2}(z, t)\right\rangle, \\
\min _{z \in[-k, k]} \epsilon_{0 k}\left[\frac{\min _{z \in[-k, k]} \hat{\tau}(z, 0)}{\min _{z \in[-k, k]} \hat{\tau}(z, 0)+(\varrho-\alpha) k}\right]^{\varrho} \leq \epsilon(z, t) .
\end{gathered}
$$

The norms of $\left\langle w_{k}^{2}\right\rangle, \epsilon_{k}$ in the space $C^{2+\alpha, 1+\alpha}\left(Q_{k}\right)$ are bounded uniformly in $k$ too, this fact follows from Schauder's estimates for linear parabolic equations. Indeed, considering equation (5.4) as a linear parabolic equation in $\left\langle w_{k}^{2}\right\rangle$ and turning to Schauder's estimates we find out

$$
\left\|\left\langle w_{k}^{2}\right\rangle\right\|_{C^{2+\alpha, 1+\alpha}} \leq c_{w}
$$

where $c_{w}$ is a constant independent of $k$. Similarly we can estimate the function $\epsilon_{k}$ in the norm of space $C^{2+\alpha, 1+\alpha / 2}$.

Thus, the sets $\left\{\left\langle w_{k}^{2}\right\rangle\right\}$ and $\left\{\epsilon_{k}\right\}$ are compact in the space $C^{2,1}$. Consequently, we can construct sequences $\left\{\left\langle w_{k_{i}}^{2}\right\rangle\right\}$ and $\left\{\epsilon_{k_{i}}\right\}$ that converge to the functions $\left\langle w^{2}\right\rangle, \epsilon$ in the norm of the space $C^{2,1}(Q)$ for each compact subset $Q \subset R_{+}^{2}$. After passage to the limit in the system (5.4), (5.5) and initial-boundary conditions (5.6), (5.7) as $k_{i} \rightarrow \infty$, we infer that $\left\langle w^{2}\right\rangle, \epsilon$ is a classical solution to the Cauchy problem:

$$
\begin{gathered}
\frac{\partial\left\langle w^{2}\right\rangle}{\partial t}=\frac{\partial}{\partial z}\left[\delta \psi(\hat{\tau}(z, t))\left\langle w^{2}(z, t)\right\rangle \frac{\partial\left\langle w^{2}\right\rangle}{\partial z}\right]-\alpha \frac{\left\langle w^{2}\right\rangle}{\psi(\hat{\tau}(z, t))}, \\
\frac{\partial \epsilon}{\partial t}=\frac{\partial}{\partial z}\left[\delta \psi\left(\hat{\tau}(z, t)\left\langle w^{2}(z, t)\right)\right\rangle \frac{\partial \epsilon}{\partial z}\right]-\varrho \frac{\epsilon}{\psi(\hat{\tau}(z, t))}, \\
\left\langle w^{2}(z, 0)\right\rangle=\left\langle w_{0}^{2}(z)\right\rangle, \quad \epsilon(z, 0)=\epsilon_{0}(z) .
\end{gathered}
$$

The function $\psi(s)$ in the system of equations (5.8), (5.9) equals $s$ by construction, since $d \leq \hat{\tau}$. To prove the preceding inequality, it suffices to apply the maximum principle to the function $\hat{\tau}$ which satisfies the equation

$$
\frac{\partial \hat{\tau}}{\partial t}=\delta\left\langle w^{2}\right\rangle \hat{\tau} \frac{\partial^{2} \hat{\tau}}{\partial z^{2}}-\alpha+\varrho
$$

Thus we have proved the following solvability theorem for problem (5.1)-(5.3): 
Theorem 5.1 Assume that $\left\langle w_{0}^{2}(z)\right\rangle, \epsilon_{0}(z)$ are continuous positive functions defined on the real axis $R$ and such that $\lim _{t \rightarrow \pm \infty}\left\langle w_{0}^{2}(z)\right\rangle=b_{ \pm}, \lim _{t \rightarrow \pm \infty} \epsilon_{0}(z)=a_{ \pm}$with finite positive numbers $a_{ \pm}, b_{ \pm}$. Then there exist a unique classical positive solution to (5.1)-(5.3).

To study the convergence of the solutions to our second-order model of turbulence to the selfsimilar solution of the third-order model we introduce into consideration the rescale variables connected by the selfsimilar description of the problem:

$$
\begin{gathered}
\varpi(\xi, \theta)=\left(t+t_{0}\right)^{2 \mu}\left\langle w^{2}(z, t)\right\rangle, \quad \chi(\xi, \theta)=\left(t+t_{0}\right)^{3 \mu}\left\langle w^{3}(z, t)\right\rangle, \\
u(\xi, \theta)=\left(t+t_{0}\right)^{2 \mu+1} \epsilon(z, t), \quad w(\xi, \theta)=\left(t+t_{0}\right)^{-1} \tilde{\tau}(z, t),
\end{gathered}
$$

where $\theta=\ln \left(t+t_{0}\right)$ is the rescaled time variable and $\mu=\alpha / 2(\varrho-\alpha)$. The functions $u, \varpi$ satisfy the system:

$$
\begin{aligned}
& \frac{\partial \varpi}{\partial \theta}=\frac{\partial}{\partial \xi}\left(\delta w \varpi \frac{\partial \varpi}{\partial \xi}\right)+(1-\mu)\left(\xi+\lambda_{0}\right) \frac{\partial \varpi}{\partial \xi} \\
& \frac{\partial u}{\partial \theta}=\delta \frac{\partial}{\partial \xi}\left(w^{2} u \frac{\partial u}{\partial \xi}\right)+(1-\mu)\left(\xi+\lambda_{0}\right) \frac{\partial u}{\partial \xi}
\end{aligned}
$$

Then $w$ is a solution of

$$
\frac{\partial w}{\partial \theta}=\delta \varpi w \frac{\partial^{2} w}{\partial \xi^{2}}+(1-\mu)\left(\xi+\lambda_{0}\right) \frac{\partial w}{\partial \xi}-w-\alpha+\varrho,
$$

and as it can be easily seen, equations (5.11)-(5.13) have a unique equilibrium state $h, f$, $w_{c}$. Here the function $h(\xi)$ is the unique solution of (4.7)-(4.8) and $w_{c}=\lim _{\theta \rightarrow \infty} w(\cdot, \theta)$, $w_{c}=\varrho-\alpha$. Therefore, any results on time asymptotic of the functions $u, \varpi, w$ translates into a result on convergence to the selfsimilar solution $\left\langle w_{a}^{2}\right\rangle,\left\langle w_{a}^{3}\right\rangle, \epsilon_{a}$.

To study the convergence of $\varpi(\xi, \theta), u(\xi, \theta)$ to a positive steady-state solution as $\theta \rightarrow \infty$, we consider the so-called limit system:

$$
\begin{aligned}
& \frac{\partial \varpi_{c}}{\partial \theta}=\partial \xi\left(\delta w_{c} \varpi \frac{\partial \varpi}{\partial \xi}\right)+(1-\mu)\left(\xi+\lambda_{0}\right) \frac{\partial \varpi_{c}}{\partial \xi} \\
& \frac{\partial u_{c}}{\partial \theta}=\delta \frac{\partial}{\partial \xi}\left(w_{c}^{2} u_{c} \frac{\partial u_{c}}{\partial \xi}\right)+(1-\mu)\left(\xi+\lambda_{0}\right) \frac{\partial u_{c}}{\partial \xi} .
\end{aligned}
$$

The initial conditions for equations (5.14), (5.15) are supposed to be the same as for $u$ and $\varpi$. The proof of the existence of positive classical solutions $u_{c}$ and $\varpi_{c}$ to the corresponding Cauchy problems is standard in Parabolic Equations Theory, see [17]. Once the existence has been established, the results of Parabolic Equations Theory yield

$$
\left|u(\xi, \theta)-u_{c}(\xi, \theta)\right| \rightarrow 0
$$

uniformly in $R$ as $\theta \rightarrow \infty$. Hence, instead of direct asymptotic investigation for system (5.11)-(5.13), we can study the asymptotic decay towards its equilibrium states of solutions to the equations for $u_{c}$ and $\varpi_{c}$. The main advantage in working with the functions $u_{c}(\xi, \theta)$ 
and $\varpi_{c}(\xi, \theta)$ is that there exist results wherein the convergence towards equilibrium has been studied in detail, in particular for equations having a form similar to (5.14), (5.15), see for example [18]. These results guarantee that

$$
u_{c}(\xi, \theta) \rightarrow h(\xi) \quad \text { and } \quad \varpi_{c}(\xi, \theta) \rightarrow f(\xi)
$$

uniformly in $R$ as $\theta \rightarrow \infty$.

Thus, the following theorem is valid:

Theorem 5.2 Solution $\left\langle w^{2}\right\rangle$, $\epsilon$ of the model (5.1)-(5.3) behaves asymptotically like $\left\langle w_{a}^{2}\right\rangle$, $\epsilon_{a}$ as $t \rightarrow \infty$.

Acknowledgments. This research was partially supported by the Russian Foundation of Basic Research (grant no. 98-01-00719). This work was supported by INTAS (proposal no. $97-2022)$

\section{References}

[1] Yanenko, N. N. (1991) Selected Works. Nauka, Moscow (in Russian).

[2] Sidorov, A. F.; Shapeev, V. P.; Yanenko, N. N. (1984) The Differential Constraints Method and its Application to Gas Dynamics. Nauka, Novosibirsk (in Russian).

[3] Ilyushin, B. B. (2000) "Higher-moment diffusion in stable stratification", in: B.E. Launder \& N.D. Sandham (Eds.) Closure Strategies for Turbulent and Transition Flows, Cambridge University Press (in press).

[4] Ilyushin, B. B. (1999) "Model of fourth-order cumulants for prediction of turbulent transport by large-scale vortex structure", J. Appl. Mechan. Tech. Phys. 40(5): 871876.

[5] Lamb, R. G. (1982) "Diffusion in convective boundary layer", in: F.T.M. Nieuwstadt \& H. van Dop (Eds.) Atmospheric Turbulence and Air Pollution Modelling, Reidel, Boston MS.

[6] Hazen, A. M. (1963) "To nonlinear theory of appearance of turbulence", Dokl. Acad. Nauk USSR 163: 1282-1287 (in Russian).

[7] Ogura, Y. (1962) "Energy transfer in a normally distributed and isotropic turbulent velocity field in two dimensions", Phys. Fluids 5(4): 395-401.

[8] Hanjalic, K.; Launder, B. E. (1972) "A Reynolds stress model of turbulence and its application to thin shear flows", J. Fluid Mech. 52: 609-638.

[9] Ibragimov, N. H. (1985) Transformation Groups Applied to Mathematical Physics. Reidel.

[10] Kaptsov, O. V. (1995) "B-determining equations: applications to nonlinear partial differential equations", Euro. J. Appl. Math., 6: 265-286. 
[11] Hulshof ,J. (1997) "Selfsimilar solutions of Barenblatt's model for turbulence", SIAM J. Math. Anal. Appl. 28(1): 33-48.

[12] Barenblatt, G. I. (1996) Scaling, Selfsimilar and Intermediate Asymptotics. Cambridge Texts in Applied Mathematics, 14.

[13] Polubarinova-Kochina, P. Ya. (1948) "On a nonlinear partial differential equation appearing in the filtration theory", Dokl. Acad. Nauk USSR, 63(6): 623-626 (in Russian).

[14] Ilyushin, B. B.; Kurbatskii, A.F. (1990) "Numerical simulation of the shearless turbulence mixing layer", Izv. Acad. Nauk USSR (Siberian Branch), Ser. Tech. Nauk. 3: $62-68$ (in Russian).

[15] Veeravalli, S.; Warhaft, Z. (1989) "The shearless turbulence mixing layer", J. Fluid Mech. 207: 191-229.

[16] Grebenev, V. N. (1994) "On a certain system of degenerate parabolic equations which arises in Hydrodynamics", Sib. Math. J. 35(4): 753-767.

[17] Ladyzhenskaya, O. A.; Solonnikov, V.; Ural'ceva N. (1968) Linear and Quasilinear Equations of Parabolic Type. Amer. Math. Soc.

[18] Vishnevskiy, M. P.; Zelenyak, T. I.; Lavrentjev, M. M. (1995) "Large time behaviour solutions for nonlinear parabolic equations", Sib. Math. J. 36(3): 510-530. 